\title{
Electronically Tunable Minimum Component Biquadratic Filters for Interface Circuits
}

\author{
Mehmet Sağbaș \\ Department of Electronics Engineering, Maltepe University, Istanbul, Turkey \\ E-mail:sagbas@maltepe.edu.tr \\ Received November 23, 2010; revised May 24, 2011; accepted June 1, 2011
}

\begin{abstract}
In this paper, two new electronically tunable filter configurations are proposed. The proposed filters operate current-mode (CM), voltage-mode (VM), transimpedance-mode (TIM) and transadmittance-mode (TAM). The first configuration realizes second-order VM band-pass and TAM high-pass filter characteristics from the same configuration. The second one realizes second-order TIM band-pass and CM low-pass filter characteristics from the same configuration. They also use minimum number of electronic components (two capacitors and one active component namely; current controlled current difference transconductance amplifier). The workability of the proposed structures has been demonstrated by simulation results.
\end{abstract}

Keywords: Second-Order Filters, CC-CDBA, Electronic Tunability, Current-Mode Circuits, Interface Circuits

\section{Introduction}

It is well known that current-mode and voltage-mode are still important integrated circuit (IC) operations [1-3]. Recently, there is a growing interest in transimpedancemode and transadmittance-mode operations. A currentinput voltage-output filter or voltage-input current-output filter is described as an interface circuit connecting a current-mode circuit to a voltage-mode circuit or a voltage-mode circuit to a current-mode circuit, respectively. These interface circuits are needed in many applications where VM and CM circuits are used together. In addition, the other important application area of transadmittancemode filters are the receiver baseband blocks of modern radio systems [4,5]. Also the outputs of the many digital/analog converters (DACs) are available as current signals. Then the transimpedance-mode filters can be used for conversion of the signals at the outputs of these DACs, simultaneously. Therefore, several TAM- and TIM-type filters are proposed using different-type active components [6-12].

Simplicity, cost reduction, power consumption and versatility are all important for the integrated circuit manufacturers. Therefore, number of the components is an important parameter. Therefore, numerous circuits are proposed in literature that employing minimum number of component [13-17]. However, these filters use at least four electronic components. The proposed filter is compared to the other filters reported in the literature by the use of Table 1. According to Table 1, the proposed filter has advantages over the proposed filters in Ref [13-14], since it has electronically tunability property and no external resistors.

In this paper, two new second order filter configurations using only single active component and two capacitors are presented. They realize CM, VM, TIM and TAM second order filter characteristics from the same configuration. Similar kinds of circuits in the literature use more than three elements [13-16] (see Table 1).

The paper is organized as in the following sections: In the next section, after a short introduction of CC-CDBA,

Table 1. Comparison of the cited references and the proposed filter.

\begin{tabular}{ccccc}
\hline Ref. & $\begin{array}{c}\text { Active } \\
\text { Element }\end{array}$ & $\begin{array}{c}\text { External } \\
\text { Capacitor }\end{array}$ & $\begin{array}{c}\text { External } \\
\text { Resistor }\end{array}$ & $\begin{array}{c}\text { Electronic } \\
\text { Tunability }\end{array}$ \\
\hline$[13]$ & 1 CDBA & 2 & 2 & No \\
{$[14]$} & 1 CDBA & 2 & 2 & No \\
{$[15]$} & 1 CCII+ & 2 & 2 & No \\
{$[16]$} & 1 CCCII & 2 & 1 & Yes \\
$\begin{array}{c}\text { Proposed } \\
\text { Filter }\end{array}$ & 1 CC-CDBA & 2 & 0 & Yes \\
\hline
\end{tabular}


two new filter configurations using CC-CDBA with two capacitors are introduced. Sensitivities and simulation results are discussed in Section 3.

\section{Proposed Resistorless Circuit Configurations and Their analysis}

In order to accomplish electronic adjustability in CDBA, Maheshwari and Khan have introduced current controlled current controlled differencing buffered amplifier (CC-CDBA) [17]. It has proven to be useful in many voltage-mode and current-mode analog signal-processing applications [17-21]. The circuit symbol of CC-CDBA is shown in Figure 1 and its terminal equation can be written as follow

$$
V_{p}=R_{p} i_{p}, V_{n}=R_{n} i_{n}, i_{z}=i_{p}-i_{n}, v_{w}=v_{z}
$$

Current controlled CDBA can easily be implemented using bipolar junction transistor (BJT) technologies shown in Figure 2 [17]. The parasitic input resistances $R_{p}$ and $R_{n}$ using BJT implementation for $I_{p, n}(t) \ll 2 I_{o}$ can be obtained as

$$
R_{p}=R_{n}=\frac{k T / q}{2 I_{O}}=\frac{V_{T}}{2 I_{O}}
$$

where, $k$ is the Boltzman's constant, $T$ is the temperature in Calvin and $q$ is the electron charge; $V_{T}=k T / q$ is the thermal voltage. Hence, $R_{p}$ and $R_{n}$ can be controlled by varying the bias current $I_{o}$. In addition to this, the quality factor $Q$ and the undamped natural frequency $\omega_{o}$ depend on $R_{p}$ and $R_{n}$, which makes them electronically adjustable.

Taking the non-idealities of CDBA into account, the above terminal equations can be rewritten as

$$
V_{p}=R_{p} i_{p}, V_{n}=R_{n} i_{n}, i_{z}=\alpha_{p} i_{p}-\alpha_{n} i_{n}, V_{w}=\beta V_{z}
$$

where $\alpha_{p}, \alpha_{n}$ and $\beta$ are the current and voltage gains, respectively, and can be expressed as $\alpha_{p}=1-\varepsilon_{p}, \alpha_{n}=1-$ $\varepsilon_{n}, \alpha_{\beta}=1-\varepsilon_{v}$, with $\left|\varepsilon_{p}\right| \ll 1,\left|\varepsilon_{n}\right| \ll 1,\left|\varepsilon_{v}\right| \ll 1 . \varepsilon_{p}$ and $\varepsilon_{n}$ denote the current tracking errors and $\varepsilon_{v}$ denotes voltage tracking error.

The proposed voltage-mode second-order band-pass filter circuit is shown in Figure 3(a). Routine analysis yields the voltage transfer function as follows:

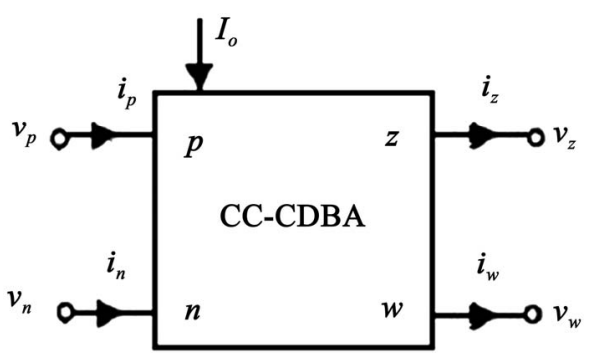

Figure 1. Block diagram of CC-CDBA.

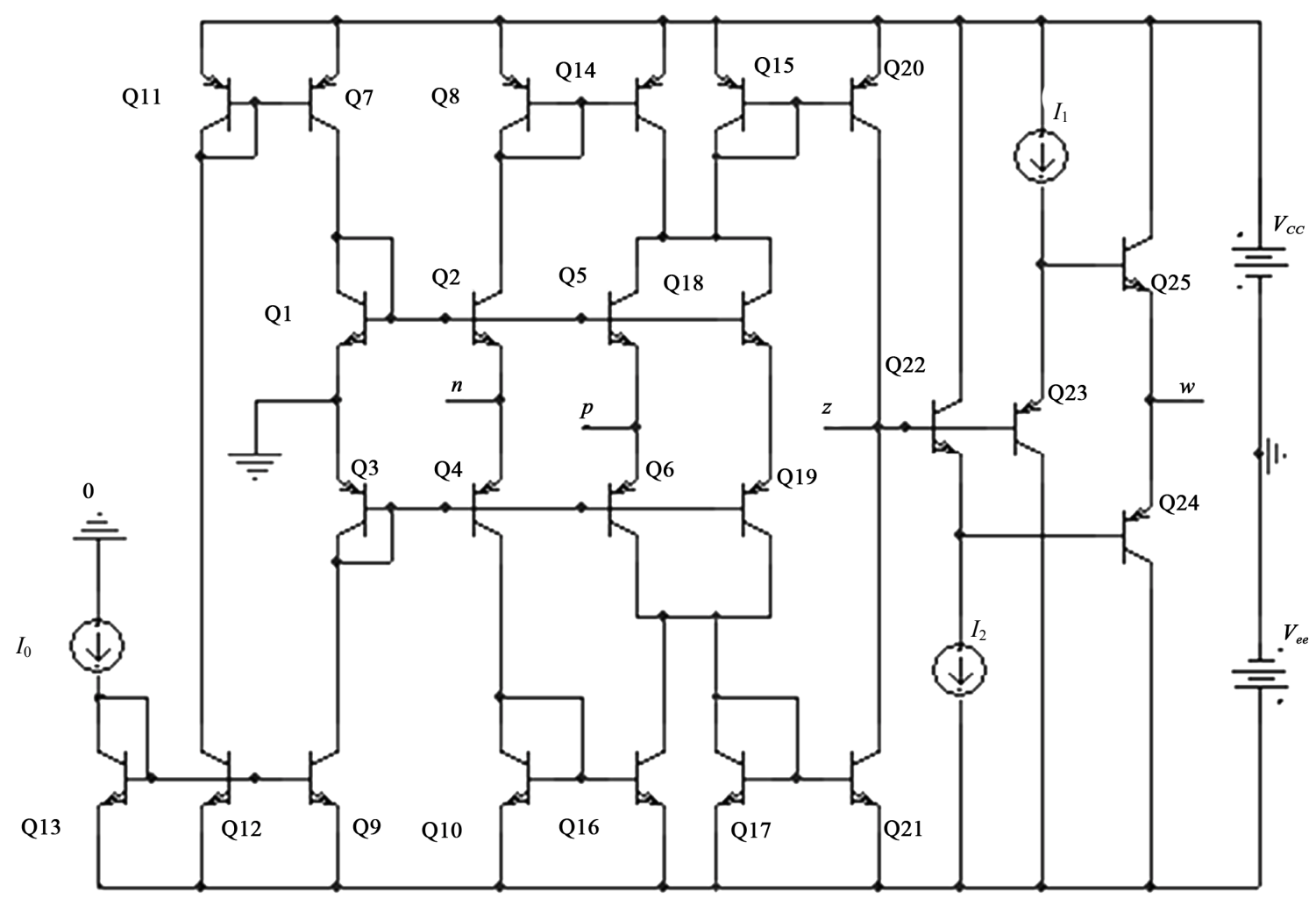

Figure 2. Schematic implementation for CC-CDBA using BJT technology. 


$$
\frac{V_{\text {out }}}{V_{\text {in }}}=\frac{s C_{1} R_{n}}{s^{2} C_{1} C_{2} R_{p} R_{n}+s\left(C_{1} R_{p}+C_{2} R_{n}\right)+1}
$$

The proposed filter in shown Figure 3(a) also gives minimum component transimpedance-mode high-pass filter response. Therefore, the current output response of the proposed circuit is

$$
\frac{I_{\text {out }}}{V_{\text {in }}}=\frac{s^{2} C_{1} C_{2} R_{n}}{s^{2} C_{1} C_{2} R_{p} R_{n}+s\left(C_{1} R_{p}+C_{2} R_{n}\right)+1}
$$

The proposed current-mode second-order band-pass filter circuit is shown in Figure 3(b). Routine analysis yields the voltage transfer function as follows:

$$
\frac{I_{\text {out }}}{I_{\text {in }}}=\frac{s C_{2} R_{n}}{s^{2} C_{1} C_{2} R_{p} R_{n}+s\left(C_{1} R_{p}+C_{2} R_{n}\right)+1}
$$

It also gives minimum component TIM low-pass filter response. Therefore, the voltage output response of the proposed circuit is

$$
\frac{V_{\text {out }}}{I_{\text {in }}}=\frac{R_{n}}{s^{2} C_{1} C_{2} R_{p} R_{n}+s\left(C_{1} R_{p}+C_{2} R_{n}\right)+1}
$$

The undamped natural frequency and the quality factor of the proposed circuit are obtained from the denominator of the transfer function as follows:

$$
\omega_{o}=\frac{1}{\sqrt{C_{1} C_{2} R_{p} R_{n}}}, \quad Q=\frac{\sqrt{C_{1} C_{2} R_{p} R_{n}}}{C_{1} R_{p}+C_{2} R_{n}}
$$

Taking the non-idealities of CC-CDBA given in Equation (3) into account, the denominator polynomial of the transfer function for the proposed filters becomes

$$
D(s)=s^{2} C_{1} C_{2} R_{p} R_{n}+s\left(\beta \alpha_{n} C_{1} R_{p}+C_{2} R_{n}\right)+\beta \alpha_{n}
$$

Using Equation (9), non-ideal the undamped natural frequency and the quality factor becomes

$$
\omega_{o}=\sqrt{\frac{\beta \alpha_{n}}{C_{l} C_{2} R_{p} R_{n}}}, \quad Q=\frac{\sqrt{\beta \alpha_{n} C_{l} C_{2} R_{p} R_{n}}}{\beta \alpha_{n} C_{l} R_{p}+C_{2} R_{n}}
$$

From Equation (10), the quality factor $Q$ and the un-

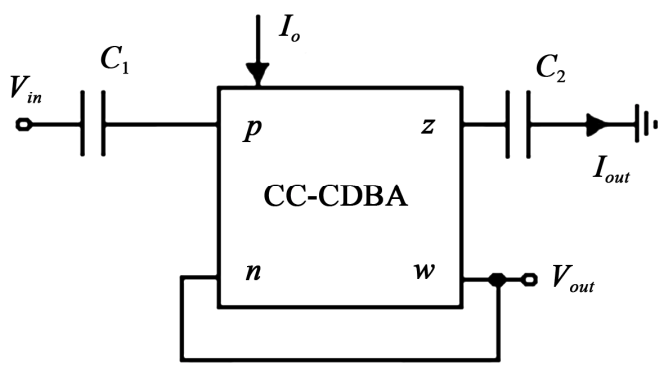

(a) damped natural frequency $\omega_{o}$ depend on $R_{p}$ and $R_{n}$ which can be controlled by varying the bias current $I_{o}$. Therefore, they can be adjusted electronically.

\section{Sensitivity Consideration and Simulation Results}

The ideal sensitivities of the natural frequency and the quality factor with respect to passive components are calculated as follows

$$
\begin{aligned}
& S_{R_{p}}^{\omega_{o}}=S_{R_{n}}^{\omega_{o}}=S_{C_{1}}^{\omega_{o}}=S_{C_{2}}^{\omega_{o}}=-0.5, \\
& S_{R_{p}}^{Q}=S_{C_{1}}^{Q}=0.5-C_{1} R_{p} k \\
& S_{R_{n}}^{Q}=S_{C_{2}}^{Q}=0.5-C_{2} R_{n} k
\end{aligned}
$$

where, $k=1 /\left(C_{1} R_{p}+C_{2} R_{n}\right)$.

If the passive component values are chosen appropriately, the ideal sensitivities will be smaller than 1 .

Using Equation (10), the non-ideal sensitivities can be found as

$$
\begin{gathered}
S_{a_{n}}^{\omega_{o}}=S_{\beta}^{\omega_{o}}=0.5, S_{a_{p}}^{\omega_{o}}=0 \\
S_{\alpha_{n}}^{Q}=S_{\beta}^{Q}=0.5-\beta \alpha_{n} C_{l} R_{p} k
\end{gathered}
$$

where, $k=1 /\left(\beta \alpha_{n} C_{1} R_{p}+C_{2} R_{n}\right)$.

Again, if passive component values are chosen appropriately, the sensitivities due to non-ideal effects will also be small than 1 .

The performance of the filter topology given in Figure 3(a) is verified using PSpice. Each CC-CDBA is realized by its BJT implementation shown in Figure 2 with the transistor model of PR100N (PNP) and NR100N (NPN) of the bipolar arrays ALA400 from AT \& T [22]. In all of the simulations, the voltage supplies of CC-CDBA are taken as $V_{c c}=2.5 \mathrm{~V}$ and $V_{e e}=-2.5 \mathrm{~V}$.

To confirm the obtained results with the theoretical results and demonstrate tunability property of the proposed configuration, the gain characteristics obtained by PSPICE for two cases are plotted in Figure 4 together. In these simulations, bias currents of CC-CDBA are $I_{o}=10$ $\mu \mathrm{A}$ and $I_{o}=20 \mu \mathrm{A}$, for simulation 1 and 2 , respectively.

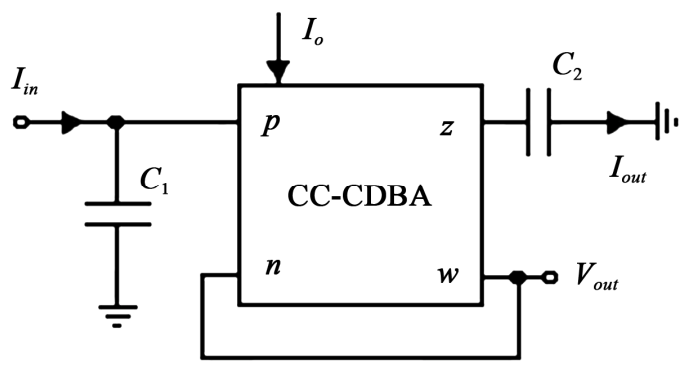

(b)

Figure 3. Circuit diagram of the proposed filters. (a) VM and TAM filter; (b) CM and TIM filter. 
For these simulations, the passive components are taken as $C_{1}=C_{2}=1 \mathrm{nF}$. These parameters correspond to a BP filter with the with the center frequency $f_{o}=124.34 \mathrm{kHz}$ and $f_{o}=248.68 \mathrm{kHz}$, quality factor $Q=0.5$, which are found by using Equation (2) (with $V_{T}=25.5 \mathrm{mV}$ thermal voltage at $25^{\circ} \mathrm{C}$ ) to find $R_{p}$ and $R_{n}$, and then Equation (8). The simulation results for the voltage-mode band-pass filter shown in Figure 4.

From the results predicted by this figure, it is concluded that the simulation results are in good agreement with the theoretical ones over a wide range of frequencies. Although, the two characteristics well coincide over a wide range of frequency, the numerical data reveal the following differences; The maximum peak attenuations for simulation I are $-6.41 \mathrm{~dB}$ and $-6.02 \mathrm{~dB}$, the maximum peak attenuations for simulation II are $6.76 \mathrm{~dB}$ and $-6.02 \mathrm{~dB}$, the center frequencies for simulation I are $117.34 \mathrm{kHz}$ and $124.34 \mathrm{kHz}$, the center frequencies for simulation II are $224.78 \mathrm{kHz}$ and $248.68 \mathrm{kHz}$ for simulation and theoretical results, respectively.

Figure 4 also shows that the dependence of the center frequency on the bias current of CC-CDBA is as predicted theoretically; namely when the bias current increases two times its tuning effect appears increasing the center frequency two times.

In order to demonstrate workability of the other output responses, the simulations are also done. For these simulation, the bias currents of CC-CDBA are taken as $I_{o}=$

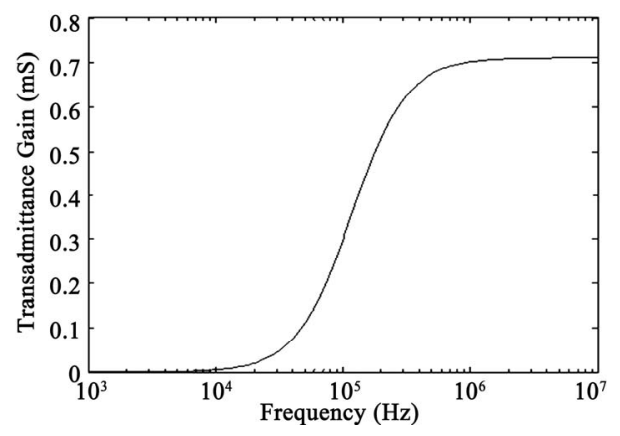

(a)
$10 \mu \mathrm{A}\left(R_{p}=R_{n}=1.3 \mathrm{k} \Omega\right)$ and the passive components are taken as $C_{1}=C_{2}=1 \mathrm{nF}$. The magnitude characteristics of the filters which are shown in Figures 3(a) and 3(b) are given in Figure 5.

\section{Conclusions}

In this paper, an electronically tunable VM band-pass, CM band-pass, TAM high-pass and TIM low-pass filters using current controlled CDBA are proposed. The proposed circuit offers the following advantageous features: 1) use of minimum number of electronic active and passive ele-

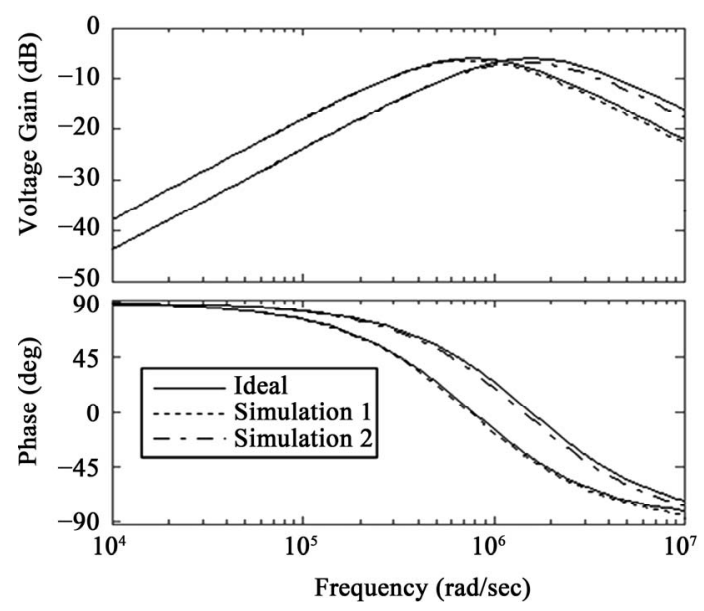

Figure 4. Simulation results for the proposed filter.

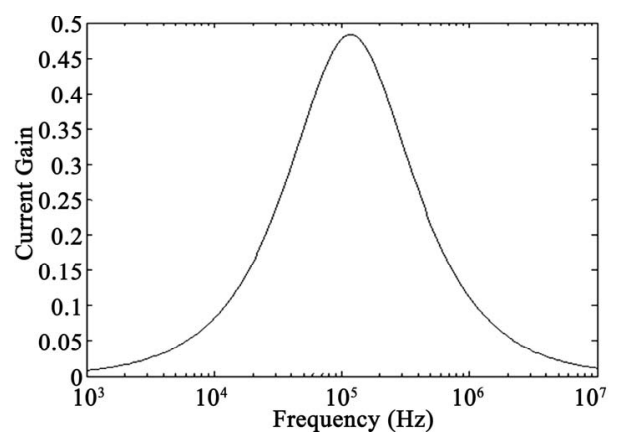

(b)

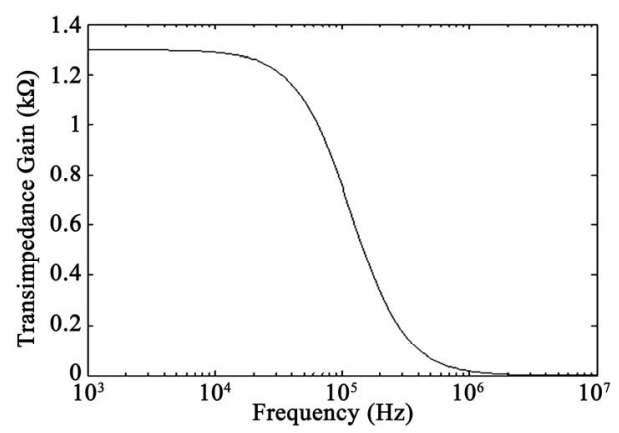

(c)

Figure 5. (a) The transadmittance grain for the proposed circuit in Figure 3(a); (b) The current gain for the proposed circuit in Figure 3(b); (c) The transimpedance gain for the proposed circuit in Figure 3(b). 
ments, namely; two grounded capacitors and one CCCDBA; 2) the quality factor and natural frequencies can be adjusted electronically without changing the values of the passive components; 3) single active component, which means less power consumption; 4) having one or more advantages over the proposed configurations in the literature [13-16]; 5) low sensitivities; 6) TIM and TAM outputs, this eliminates the need for current to voltage or voltage to current conversions in DAC and ADC applications; The above properties most of which are well verified by the PSpice simulation make the proposed filter attractive for circuit designers and engineers.

\section{References}

[1] B. Wilson, "Recent Developments in Current Conveyors and Current-Mode Circuits," IEE Proceedings of Circuits, Devices and Systems, Vol. 137, No. 2, 1990, pp. 63-77.

[2] D. C. Wandsworth, "Accurate Current Conveyor Integrated Circuits," Electronics Letters, Vol. 25, No. 18, 1989, p. 1251. doi:10.1049/el:19890839

[3] G. Gilbert, "Current Mode, Voltage Mode, or Free Mode? A Few Sage Suggestions," Analog Integrated Circuits and Signal Processing, Vol. 38, No. 2-3, 2004, pp. 83-101. doi:10.1023/B:ALOG.0000011161.44537.da

[4] G. W. Rundell, J. J. Ou, T. B. Cho, G. Chien, F. Brianti, J. A. Weldon and P. Grey, "A 1.9-Ghz Wide-Band IF Double Conversion CMOS Receiver for Cordless Telephone Applications," IEEE Journal of Solid-State Circuits, Vol. 32, No. 12, 1997, pp. 2071-2088. doi:10.1109/4.643665

[5] M. S. Steyaert, J. Janssens, B. D. Muer, M. Borremans and N. Itoh, "A 2-V CMOS Cellular Transceiver FrontEnd," IEEE Journal of Solid-State Circuits, Vol. 35, No. 12, 2000, pp. 1895-1907. doi:10.1109/4.890303

[6] S. Minaei, G. Topcu and O. Cicekoglu, "Low Input Impedance Trans-impedance Type Multifunction Filter Using Only Active Elements," International Journal of Electronics, Vol. 92, No. 7, 2005, pp. 385-392. doi:10.1080/00207210412331334789

[7] A. Toker, O. Cicekoglu, S. Ozcan and H. Kuntman, "HighOutput-Impedance Transadmittance Type ContinuousTime Multifunction Filter with Minimum Active Elements," International Journal of Electronics, Vol. 88, No. 10, 2001, pp. 1085-1091. doi:10.1080/00207210110071260

[8] U. Cam, "A New Transadmittance Type First-Order Allpass Filter Employing Single Third Generation Current Conveyor," Analog Integrated Circuits and Signal Processing, Vol. 43, No. 1, 2005, pp. 97-99. doi:10.1007/s10470-005-6576-9

[9] N. A. Shah, S. Z. Iqbal and B. Parveen, "SITO High Output Impedance Transadmittance Filter Using FTFNs," Analog Integrated Circuits and Signal Processing, Vol. 40, No. 1, 2004, pp. 87-89. doi:10.1023/B:ALOG.0000031438.72455.b1

[10] U. Cam, C. Cakir and O. Cicekoglu, "Novel Transimpe- dance Type First-Order All-Pass Filter Using Single OTRA," International Journal of Electronics and Communication, Vol. 58, No. 4, 2004, pp. 296-298. doi:10.1078/1434-8411-54100246

[11] M. Sagbas and M. Koksal, "Voltage-Mode Three-Input Single-Output Multifunction Filters Employing Minimum Number of Components," Frequenz, Vol. 61, No. 3-4, 2007, pp. 87-93. doi:10.1515/FREQ.2007.61.3-4.87

[12] M. Sagbas and M. Koksal, "Current-Mode State-Variable Filter," Frequenz, Vol. 62, No. 1-2, 2008, pp. 37-42. doi:10.1515/FREQ.2008.62.1-2.37

[13] A. U. Keskin, "Voltage-Mode High- $Q$ Band-Pass Filters and Oscillators Employing Single CDBA and Minimum Number of Components," International Journal of Electronics, Vol. 92, No. 8, 2005, pp. 479-487. doi: $10.1080 / 00207210500168626$

[14] U. Cam, "A Novel Current-Mode Second-Order Notch Filter Configuration Employing Single CDBA and Reduced Number of Passive Components," Computers and Electrical Engineering, Vol. 30, No. 2, 2004, pp. 147-151. doi:10.1016/S0045-7906(03)00002-8

[15] S. Özcan, H. Kuntman and O. Cicekoglu, "Multi-input Single-Output Filter with Reduced Number of Passive Elements Using Single Current Conveyor," Computers and Electrical Engineering, Vol. 29, No. 1, 2003, pp. 45-53. doi:10.1016/S0045-7906(01)00024-6

[16] S. Minaei, O. Cicekoglu, H. Kuntman and S. Turkoz, "High Output İmpedance Current-Mode Lowpass, Bandpass and Highpass Filters Using Current Controlled Conveyors," International Journal of Electronics, Vol. 88, No. 8, 2001, pp. 915-922. doi: $10.1080 / 00207210110058166$

[17] S. Maheshwari and I. A. Khan, "Current Controlled Current Differencing Buffered Amplifier: Implementation and Applications," Active and Passive Electronics Components, Vol. 4, No. 4, 2004, pp. 219-227. doi:10.1080/08827510310001648924

[18] M. Koksal and M. Sagbas, "General Synthesis Procedure for $n^{\text {th }}$-Order Current Transfer Function Using CDBA," Frequenz, Vol. 61, No. 3-4, 2007, pp. 94-101. doi:10.1515/FREQ.2007.61.3-4.94

[19] M. Koksal, M. Sagbas and H. Sedef, "An Electronically Tunable Oscillator Using a Single Active Device and Two Capacitors," Vol. 17, No. 5, 2008, pp. 885-891.

[20] P. Silapan, W. Jaikla and M. Siripruchyanun, "High-Performance BiCMOS Current Controlled CDBA and Application," International Symposium on Communication and Information Technology, Vol. 1-3, 2007, pp. 40-43.

[21] W. Tangsrirat, D. Prasertsom and W. Surakampontorn, "Low-Voltage Digitally Controlled Current Differencing Buffered Amplifier and Its Application," AEU-International Journal of Electronics and Communications, Vol. 63, No. 4, 2009, pp. 249-258. doi:10.1016/j.aeue.2008.01.006

[22] D. R. Frey, "Log-Domain Filtering: An Approach to Current-Mode Filtering," IEE Proceedings-G: Circuits, Devices and Systems, Vol. 140, No. 6, 1993, 406-416. doi:10.1049/ip-g-2.1993.0066 